\title{
Linx
}

Revue des linguistes de l'université Paris X Nanterre

$12 \mid 2002$

"Comme la lettre dit la vie »

\section{Des « profileurs » de l'énonciation : les constructions avec genre, sorte et espèce}

Laurence Rosier

\section{OpenEdition}

Journals

Édition électronique

URL : http://journals.openedition.org/linx/1313

DOI : $10.4000 / \operatorname{linx} .1313$

ISSN : 2118-9692

Éditeur

Presses universitaires de Paris Nanterre

Édition imprimée

Date de publication : 1 octobre 2002

Pagination : 246-253

ISSN : 0246-8743

\section{Référence électronique}

Laurence Rosier, «Des «profileurs » de l'énonciation : les constructions avec genre, sorte et espèce », Linx [En ligne], 12 | 2002, mis en ligne le 10 octobre 2012, consulté le 20 avril 2019. URL : http:// journals.openedition.org/linx/1313 ; DOI : 10.4000/linx.1313

Ce document a été généré automatiquement le 20 avril 2019

Département de Sciences du langage, Université Paris Ouest 


\title{
Des « profileurs » de l'énonciation : les constructions avec genre, sorte et espèce
}

\author{
Laurence Rosier
}

\section{Introduction}

1 Les travaux de Michelle Perret pourraient être qualifiés d'une linguistique des « situations ». Dire d'où l'on parle, d'où l'on écrit (cf. l'intérêt pour le paratexte englobant les gloses réflexives sur la narration elle-même), dire comment le texte construit son propre mode de référence situationnelle: les fines analyses des indexicaux superposent approche spécifique des mediums utilisés, syntaxe, sens et rôle performatifs des marqueurs linguistiques de situation (ce qu'on regroupe communément sous le terme embarrassant de deixis, cf. Perret 1985). La question du repérage implique de mettre en situation le dit ou l'écrit et de se préoccuper tant de l'encodage que du décodage de l'acte d'énonciation. Situer, c'est situer non seulement par rapport à soi mais pour que l'autre puisse à son tour situer.

2 C'est dans cette perspective énonciative que nous présenterons quelques usages des mots genre, espèce et sorte du point de vue de leurs emplois en "enclosure », donc en noyau d'une structure nominale (déterminant + N1 + de + N2 un/une genre/espèce/sorte de valise). Ces syntagmes ne sont pas bien entendu des morphèmes relevant de la deixis : mais ils ont l'art de présenter le dire sous un éclairage énonciatif particulier, de l'évaluer pour reprendre le traitement proposé par Yaguello (1998), de le situer, ou, plus exactement, de le profiler (cf. infra) plus largement dans le cadre de l'échange interlocutif en créant une connivence de discours.

3 L'analyse est située plus largement dans le cadre d'une approche du lexique à la fois syntaxique, sémantique et discursive, rejoignant le travail déjà présenté dans trois articles parus et à paraître (Rosier 2000, 2002). Nous y avons traité de la grammaticalisation du terme genre ainsi que des emplois contrastifs avec espèce, sorte, 
type, style, tendance, version, etc. Nous avons également mis en avant un continuum sémantique du terme genre selon les emplois grammaticaux (du nom aux enclosures, locutions prépositionnelles, particule modale cf. les travaux de Yaguello) allant de l'identification stricte d'une catégorie (les genres littéraires) à la catégorisation floue ou approximative (un genre de petite écharpe = quelque chose qui y ressemble, il est genre dix heures $=$ il est environ dix heures). C'est précisément cette approximation comme mécanisme énonciatif que nous allons appréhender dans ce petit article ${ }^{1}$.

\section{L'approximation comme évaluation}

De façon intuitive et banalisée, on pourrait dire que l'approximation n'a pas bonne presse : être approximatif, c'est ne pas toucher juste. C'est pratiquer l'à peu près. En se contentant de cette première acception, on peut lister une série de manifestations linguistiques qui exprime cette approximation, tantôt qualitative, tantôt quantitative :

1. l'emploi de comme: comparez un écrivain comme Aragon, qui permet une double lecture d'identification stricte (Aragon lui-même) ou de création d'une sous-catégorie d'écrivain dont on nous donne un exemplaire : qui a des caractéristiques communes avec Aragon) et elle est comme folle (elle se rapproche de cette catégorie).

2. la structure prépositionnelle : à la + Nom : à la garçonne paraphrasable en à la manière de. De même les syntagmes prépositionnels signifiant à la manière de : à la Monet = comme Monet.

3. la dérivation : d'allure garçonnière = qui rappelle celle d'un garçon, comme un garçon mais qui n'en est pas obligatoirement un; des accents rimbaldiens et autres compositions à partir des noms propres = des accents qui rappellent Rimbaud, comme ceux de Rimbaud, etc.

4. les enclosures: une sorte de, une espèce de connaissent la même ambivalence : ils peuvent conforter mais aussi simplement s'approcher d'une catégorie. Wilmet (1998: 229) propose comme paraphrase d'une sorte de martien : un martien qui n'en est pas tout à fait un.

5. l'adjonction à un caractérisant d'un adverbe comme plutôt, assez, un peu, quasi.

6. l'antéposition de certains adjectifs : comparer un curieux malade (est-il bien malade ? Il y a une remise en cause de la catégorie posée; le malade ne présente pas les caractéristiques attendues du malade typique), et un malade curieux (c'est un malade qui présente toutes les caractéristiques pour être appelé tel et il est curieux ou il suscite la curiosité).

7. Les surcompositions d'expression (cf. Noailly 1993) par exemple une fresque-road moviecomédie trash, symbole de l'inadéquation entre le réel et le lexique inexistant susceptible de le dire.

8. Les déterminants comme dans Deux ou trois choses que je sais d'elle, il y avait quatre cinq personnes qui attendaient : il y a là une quantification approximative.

5 Pourtant, la définition donnée par les dictionnaires est plus positive : l'approximation y est décrite comme une "évaluation approchée ». De surcroit, l'acception mathématique $\mathrm{du}$ terme fait de l'approximation un «algorithme permettant d'obtenir une solution approchée de plus en plus précise d'un problème numérique ». Or l'évaluation est au centre des théories de l'énonciation, notamment dans le cadre des réflexions issues de Bakthine (trad. 1977) :

Tout mot actualisé comporte non seulement un thème et une signification au sens objectif, de contenu, de ces termes, mais également un accent de valeur ou appréciatif, c'est-à-dire que, lorsqu'un contenu objectif est exprimé (dit ou écrit) par la parole vivante, il est toujours accompagné d'un accent appréciatif déterminé (147) 
6 L'appréciation ou l'évaluation du dire est au centre des formes citées en 4), qui retiennent ici notre attention. Si Yaguello a insisté sur l'évaluation modale de l'emploi du terme genre, nous prenons également en compte l'«évaluation approchée » exprimée par le terme et sa clique. Cette évaluation approchée concerne au premier chef une dimension définitoire, métalinguistique, qui convoque à la fois des procédés relevant de la dénomination, de la comparaison et de la métaphore. Nous allons les examiner successivement.

\section{2. L'approximation définitoire}

7 On trouve cette approche notamment chez Martin (1983), chez Pottier (1992), chez Noailly (1993). Le premier énumère les types de définition possible : métalinguistiques et/ ou paraphrastiques, celle-ci se subdivise en hyperonymiques, métonymiques, dérivationnelles et approximative. Cette dernière retient tout particulièrement notre attention :

La définition par approximation est une commodité où le lexicographe fait usage d'indicateurs du type "sorte de ", "espèce de »; ces définitions s'utilisent en désespoir de cause ; elles n'en sont pas moins une origine possible pour des énoncés analytiques (quiche « sorte de tarte de pâte brisée, garnie d'une préparation à base de crème et d'œufs » Petit Robert) (p. 58).

8 Martin note également qu'un même vocable peut se prêter à diverses formes de définition. Ainsi quiche est défini par approximation dans le Robert et dans le Dictionnaire du français contemporain par l'hyperonyme flan. De notre côté, nous avons vérifié dans le Larousse qui donne lui l'hyperonyme tarte.

Dans Sémantique générale (1992), Pottier analyse les modes de désignation immédiate et médiate : dans le premier cas, les « entités » ont une désignation privilégiée par exemple : Là où le maire de Paris a son bureau est l'hôtel de ville (p. 123). Mais il est des cas, poursuit-il, où l'énonciateur prend ses distances, rendant les opérations de désignation plus complexes: elle devient médiate. Et de citer la métonymie, la métaphore et les phénomènes de paraphrase (péronymie).

Enfin, Noailly (1993) traite des mots composés, lexicalisés (type jupe-culotte) ou non (une farce-comptine-mystère-chanson-tragi-comédie, relevé dans le Monde) et insiste sur le fait que :

Le caractère approximatif, imparfait de la description de l'objet visé est repérable au fait que la série des termes associés pourrait être précédée d'une expression qui sanctionne l'a-peu-près, une sorte de (dans certains cas, en quelque sorte) : « une sorte de farce-comptine- mystère » etc. (p. 79-80)

11 Ces trois réflexions brièvement décrites mettent en avant la possibilité d'une médiation en discours lorsqu'un terme synthétique (Pottier dirait un «orthonyme») n'est pas disponible. Le recours aux enclosures, dans leur versant approximatif, est clairement noté chez Noailly (une sorte de jupe-culotte pour signifier que ce n'est pas une jupe culotte prototypique ; cf. paraphrases identiques chez Larrivée 1994, également Wilmet 1998). La péronymie (le fait de " tourner autour ») chez Pottier s'accommode parfaitement de notre expression de l'approximation, explicitement présente comme processus définitoire chez Martin.

Cependant, les emplois sur lesquels nous travaillons doivent être mis sur une échelle de degrés car ils n'actualisent pas toujours l'approximation. 


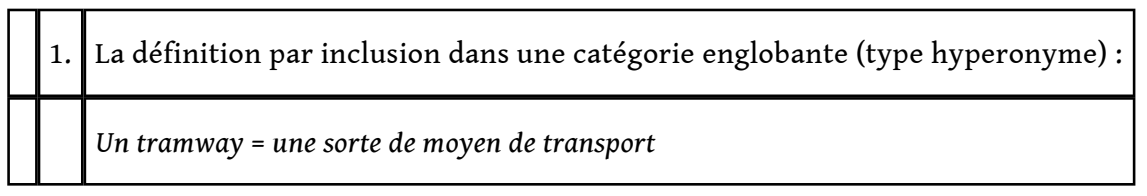

14 Cette acception n'est pas possible avec espèce et genre dans les enclosures. Si on dit « un tramway est une espèce ou un genre de moyen de transport ", c'est manifestement l'approximation qui est activée dans ce cas. Alors que sorte pousse à postuler d'office un sous-ensemble d'une catégorie clairement posée. Par exemple la baleine est une sorte de mammifere implique qu'il y a diverses sortes de mammifères par exemple, mammifere marin, mammifere ongulé, mammifère carnivore, etc. Cependant l'énonciation la plus naturelle dans ce cas est de se passer de l'enclosure : un tramway est un moyen de transport. L'exemple qui suit, avec espèce, permet pourtant une interprétation approximative, alors que l'on se trouve en présence d'une catégorie animale reconnue dans le savoir encyclopédique :

Je crois que c'est une espèce de baleine dont les naturalistes ne font point mention

(G. de Foigny, La terre australe connue 1676, p. 66)

Soit l'appartenance à la classe des baleines pourra être par la suite vérifiée, soit l'objet y ressemble mais n'est pas répertorié comme relevant de la classe. La présence d'un verbe introducteur d'attitude propositionnelle (je crois) n'est pas étrangère à cette interprétation, nous y revenons ci-dessous.

2. La définition par rapprochement parasynonymique ou peronymique

16 Un tramway = une sorte, une espèce, un genre de métro. L'expression est un parasynonyme complexe. Le second terme peut être caractérisé et on se trouve alors face à une paraphrase analytique (type : Le fils de Louis XIII et d'Anne d'Autriche = Louis XIV). Mais cette paraphrase n'a pas nécessairement de correspondant synthétique. Par exemple, dans le corpus quels seraient les termes capables de synthétiser protubérance biscornue, saut avorté, chevrotement rauque, rire immobile, plainte farouche, vraisemblance fantastique, fougue taciturne, jupe-culotte ultra longue, ultra ample, brute ou délavée.

17 Le GN [N2 + caractérisation] constitue un groupe qui pourrait être redénommé si un nom est disponible. Dans les cas qui nous retiennent, il semble justement que le nom fasse défaut et que l'approximation porte métalinguistiquement sur l'expression. Pottier (1992 : 125) insiste sur le fait que « la péronymie peut être circonstancielle et ne pas être instituée dans le savoir partagé » (p. 125), d'où la difficulté de trouver le terme subsumant l'expression complexe.

18 L'approximation dans ce cas porte essentiellement sur la dimension métalinguistique: l'évaluation approchée (un mot ou une expression qui ne vont pas de soi sont choisis « en désespoir de cause » pour reprendre l'expression de Pottier) est manifeste.

Cela faisait une sorte de banquet féminin, ce qui est une contradiction dans les termes, puisque les femmes étaient toutes debout, comme c'est la coutume, et, pour la moitié d'entre elles, à la cuisine (G. Brisac, Week-end de chasse à la mère, 1996, p. 165). 
Dans certains exemples, la présence d'une dénomination synthétique montre l'ancrage métalinguistique (cf. dans les exemples ci-dessous, par l'apposition ou par la présence du verbe appeler) de façon soit prospective (une fouine [E] rétrospective (sorte de tableau ... 監un trésor de mer):

Georges avait emprunté à son frère une fouine, une sorte de harpon à très long manche, aux pointes barbelées, que l'on utilise dans le marais pour pêcher des anguilles. (J. Rolin, L'organisation, p. 77)

Ce que j'aimerais faire, ce serait une sorte de tableau comme nous en fabriquions autrefois, nous appelions cela un trésor de mer. (Geneviève Brisac, Week-end de chasse à la mère, p. 198)

La définition par métaphore

Un tramway = une sorte de girafe obèse. Il suffit d'une association conceptuelle pour justifier ce qui constitue alors une recatégorisation et une redénomination.

Ici, on rétablit une identification puisqu'on quitte l'approximation (les deux objets ne sont pas " proches " sémantiquement) et on tend à revenir à une nouvelle dénomination analytique et non plus synthétique.

...quelquefois lorsqu'on regarde entre les guillemets qui entourent le mot culture c' est une sorte de paquet surprise ... (Corpus Orléans )

Le bailli du Palais était une espèce de magistrat amphibie, une sorte de chauvesouris de l'ordre judiciaire, tenant à la fois du rat et de l'oiseau, du juge et du soldat (Hugo, Notre-Dame de Paris, p. 79)

Il y a même une étoile du berger tout en haut comme un genre de petit chapeau pour dame. Un sapin réussi doit ressembler à un dessin d'enfant (G. Brisac, Weekend de chasse chez ma mère, p. 90).

Dans son ouvrage Le sens figuré (1981), Tamba-Mecz explique la manière dont fonctionne, $\mathrm{du}$ point de vue énonciatif, les figures : par l'analogie et la localisation spatiale (« la situation spatiale des configurations imaginaires» p. 192) elles permettent à l'énonciateur de présenter son dire comme une semblance ou une approximation. Nos exemples semblent bien pouvoir relever de ce type d'explication, où l'objet désigné n'est pas appréhendé directement car sa désignation n'appartient pas au savoir commun et est atteinte par médiation.

Pour les cas qui ressortissent à 2, c'est-à-dire où l'approximation est l'interprétation la plus partagée, espèce, genre et sorte profilent une interprétation approximative, une évaluation approchée. Mais le rôle du N2 dans l'enclosure ne fait-il pas pencher la balance sémantique d'un côté ou de l'autre? Et quel est le rôle de la prédication? Quel est également le rapport établi (inclusion ou non dans un ensemble supérieur) entre les termes en présence? En effet notre approche ne peut en aucun cas négliger le rôle du cotexte et du contexte où apparaissent les constructions. La structuration du champs sémantique de chaque N1 inclut, au terme de réglages ou de sédimentations historiques, la présence privilégiée ou quasi-exclusive de l'un des trois profileurs par rapport à N2 : par exemple, espèce concerne de façon privilégiée les catégories biologiques et chimiques ; genre s'applique à la subdivision de la classification des êtres vivants ou des catégories d'œuvres.

Mais de toutes les façons, il importe d'insister sur le fait que, dans la perspective d'une analyse énonciative, la double lecture de nos expressions est toujours possible. 


\subsection{L'approximation en cotexte et en contexte}

ens les noms en position de N2. Un rapport hyperonymique clairement reconnu dans le savoir encyclopédique occulte une interprétation approximative. Ainsi, par exemple, Le chat est une espèce de félin convient parfaitement puisque l'espèce regarde la catégorisation animale. Comme nous l'avons dit, on peut même se passer d'une étape dans ce cas et passer directement à la simple prédication: Le chat est un félin. Sorte est plus neutre de ce point de vue et admet à la fois des sous-catégories animales, végétales, humaines, etc. (exemple attesté oral du corpus Orléans : Des sortes de Français, pour parler de catégories comme les instituteurs). Quant à genre, il a tendance, parallèlement à sa grammaticalisation, à privilégier l'interprétation approximative ou exemplaire plutôt que l'identification d'un genre stricto sensu. Dans Le genre poétique, genre est un nom et la caractérisation restreint à un sous-ensemble des genres littéraires. Dans c'est un genre de poésie, où genre est le noyau de l'enclosure, la lecture est soit approximative (cela ressemble à de la poésie) soit identifiante mais de façon « décalée » (dans le genre appelé poésie, il y a de la poésie lyrique, épique, descriptive, etc.) mais on utilisera dans ce sens moins genre qui concerne des catégories plus larges que type ou sorte (pour sous-catégoriser).

Nous avons été attirée, lors du dépouillement du corpus, par le fait que les N2 pouvaient se classer selon l'opposition abstrait/concret. Nous n'avons pas fait de comptage des termes N2, en raison du nombre excessif d'occurrences données par la base de données Frantext (environ 6000 mentions pour espèce, 13000 pour sorte et seulement 700 pour genre) mais le nombre de noms abstraits dans ces expressions a tendance à dominer: miracle, caprice, bonheur, lâcheté, charme, mal, sacrifice, rage, pitié....

C'est une espèce de bonheur de connaître jusques à quel point on doit être malheureux (La Rochefoucauld, Maximes, p. 295)

Il est un peu de mes amis, et ce seroit à moi une espèce de lâcheté, que d'en oür dire du mal (Molière, Dom Juan, p. 151)

Je sais qu' il y a une espèce de contrat et même le plus saint de tous entre le bienfaiteur et l'obligé : c'est une sorte de société qu'ils forment l'un avec l'autre, plus étroite que celle qui unit les hommes (Rousseau, Les rêveries du promeneur solitaire, p. 94)

Les noms concrets se rencontrent cependant également : cercueil, fruit, jupe, baleine, bois, monticule, couronne, croix, chaloupe, noix, fruit, dragon...;

Plus j'y pensois, plus j'étois surpris, et moins je me pouvois résoudre, jusqu'à ce qu'un vaisseau françois amena au port une espèce de chaloupe très bien faite, d'une figure plus ronde qu'ovale avec deux becs d'oyseau aux deux extrémitez (G. de Foigny, La terre australe connue, 1676, p. 161).

Elle arriva enfin à une espèce de monticule couronné de vingt ou trente sapins qui formaient là comme une sorte de bois (Th. Gautier, Le capitaine Fracasse, p. 70).

Cette distinction concret/abstrait est-elle cependant pertinente ? L'abstraction est-elle liée à l'approximation? Et dans nos exemples historiques, peut-on déjà parler d'interprétation approximative (c'est-à-dire considérer nos exemples comme des enclosures) ? Vu la difficulté de définir l'intension des noms abstraits à moins de passer par un support (pour comprendre le courage, on doit passer par un homme courageux cf. théorie de Wilmet 1998) et la relativité qui en découle (suivant l'homme qu'on imagine courageux, l'image du courage est sensiblement différente), on pourrait imaginer qu'avec les noms abstraits, c'est en effet l'interprétation approximative qui l'emporte. Noailly (op. cit.) va dans le même sens à propos des dénominations analytiques contradictoires (désir- 
crainte, haine-amitié, etc.) plus courantes avec le vocabulaire abstrait qu'avec le vocabulaire concret :

Le principe d'irréductibilité référentielle n'est pas de mise ici, tout simplement, sans doute, parce qu'il n'a de pertinence que dans les cas où sont mises en contact des réalités concrètes. Le vocabulaire abstrait semble ne pas être soumis aux mêmes contraintes de la contingence [...] la libre-formation des composés abstraits ou concrets pallie de façon médiate (et parfois provisoire) les carences du lexique fondamental (p. 83)

Mais cette hypothèse devrait être étayée sur corpus de façon plus systématique.

Cependant, on peut affirmer que l'approximation portée par les enclosures est dépendante du nom avec lequel elles se construisent. En effet, la reconnaissance de catégories ontologiques suppose une interprétation identifiante: sinon l'analogique prend le pas et on verse dans l'approximation. Dans l'exemple qui vient (issu de l'oral), il est évident qu'on peut lister des sous-catégorisations (18) de la catégorie « baccalauréat » et l'exemple de Rousseau sous-entend qu'il y a plusieurs manières répertoriée de travailler la terre :

il y a je crois dix-huit sortes de baccalauréats ( Corpus Orléans)

Ce rapport dépend de la fertilité du climat, de la sorte de travail que la terre exige, de la nature de ses productions, de la force de ses habitans, de la plus ou moins grande consommation qui leur est nécessaire, et de plusieurs autres rapports semblables desquels il est composé. (Rousseau, Du contrat social, p. 282)

Du point de vue de l'analyse grammaticale, ces cas donnent la prééminence au N1 (sorte) et non pas au N2 (qui est un complément déterminatif), ce qui bloque l'interprétation approximative. Alors que dans un cas comme :

Ils sautaient dans une sorte de cabine d'ascenseur remplie de balles élastiques et semblaient tout pâles. (Geneviève Brisac, Week-end de chasse à la mère, p. 106)

c'est le N2 (cabine d'ascenseur) qui est le noyau et une sorte de est un déterminant complexe, additionnant de la caractérisation et de la quantification. Cabine d'ascenseur ne repose pas du point de vue de nos connaissances encyclopédiques sur des « sortes » ou des sous-catégorisations reconnues.

Le rôle joué par les déterminants doit également être mis en avant: en effet dans les exemples que nous venons de discuter, le déterminant un favorise clairement l'interprétation approximative, alors que le numéral et l'article défini privilégient la reconnaissance claire de catégories ou de sous-catégories².

Au-delà du N2, l'approximation s'actualise ou au contraire est bloquée dans certains cotextes énonciatifs :

\section{approximation bloquée}

- la détermination : l'emploi de une comme numéral en regard de plusieurs :

Il n'y avait pas une espèce de mal mais plusieurs (Calvin, Institution chrétienne 1560, p. 25)

36 - la prédication:

Un petit bandeau qui était une espèce de couronne (N. de Peiresc, Lettres, $1602-1637$, t2, p. 123

Le petit bandeau fait office de couronne, avec laquelle il présente des caractéristiques définitoires communes: il offre des caractéristiques permettant de le prendre fonctionnellement pour un autre objet. Le bandeau ceint la tête comme le fait une couronne. 

une énumération pour cerner à l'aide d'expressions enfilées ce qu'il est difficile de nommer immédiatement.

Même du temps de Henry III, on croyait à Paris que c'étoit une espèce de malédiction aux normands, ou plutôt de punition de ce qu' ils ne beuvoient que du sidre. (G. Patin, Lettres 1630-1649, p. 279).

Il y a une espèce de plante, moitié ronce, moitié arbrisseau, qui produit, dans des coques hérissées de pointes (Bernardin de Saint-Pierre, Voyage à l'île de France, 1773, p. 41).

On dirait une espèce de coma ou de léthargie (J. Romains, Verdun, 1938, p. 52).

...il est couvert, depuis l'estomac jusqu'aux pieds, d'une toile fine fort plissée, qui fait comme une espèce de jupe (Aulnoy, M.C., Relation du voyage d'Espagne, 1691, p. 103)

mais alors il dit ils nous servaient des des espèces de petits pains comme des petits gâteaux (Corpus Orléans)

Dans le même ordre de fait, on notera les suites espèce de + sorte de et vice-versa :alternant espèce de et sorte de, elles illustrent également une évaluation rapprochée par pallier, à la recherche du mot juste, de la bonne expression dénominatrice.

N'est-ce pas une sorte de radotage, une espèce de faiblesse d'esprit que de s'occuper de lettres dans ce moment? (Chateaubriand, Mémoires d'Outre-tombe, p. 20)

Les autres ont pensé que cette écriture était une sorte de don du hasard, une espèce de trouvaille fortuite que rien n' avait préparé (Destutt de Tracy, Elements d'idéologie gramm., 1803, p. 349)

44 - le point de vue et l'énonciation rapportée : Les éléments favorisant l'interprétation approximative regardent également l'énonciation: les enclosures figurant dans des formes de discours rapporté, principalement en discours indirect libre, après des verbes sentiendi ou d'attitude propositionnelle, nous mettent dans la conscience des personnages et favorisent la relativité de leur expérience vécue et donc l'approximation.

Anny me regardait avec une espèce de tendresse (Sartre, La Nausée, p. 174)

Quelque chose de plus aimable en la lui faisant connaître, elle était apparue à Swann non pas certes sans beauté mais d'un genre de beauté qui ne lui était pas indifférent, qui ne lui inspirait aucun désir, lui causait même une sorte de répulsion physique (Proust, Du côté de chez Swann, 1913, p. 195)

Dans Exercices de style, Queneau use habilement des enclosures dans trois textes: réactionnaire, ignorance et fantômatique. Ces exemples sont remarquables car ils illustrent 1) le point de vue du réactionnaire qui note l'inadéquation du mot et de la chose, 2) le 
point de vue de l'ignorant qui ne trouve pas le bon mot, 3) le point de vue relatif obligeant l'énonciateur à prendre ses distances : on entre dans sa vision subjective.

Cette fois-là je n'avais dans mon voisinage que des hommes, dont une sorte de zazou au cou démesuré et qui portait autour de son feutre mou une espèce de tresse au lieu de ruban (Queneau, Exercices de style, p. 130)

\section{L'approximation comme connivence}

Ces enclosures proposent donc une sorte de profil de typicalité qui doit permettre à l'interlocuteur de reconnaître de quoi on parle. Nous avions insisté précédemment (Rosier 2000) sur le rapport étalon/prototype instauré par ces constructions, d'où le terme alors proposé de rapporteur. Mais nous pensons que celui de «profileur» leur sied davantage.

La connivence est particulièrement illustrée par l'emploi des enclosures avec nom propre : soit il reste opaque (mais comme c'est du reste le cas avec tout terme difficile c'est-à-dire de faible diffusion médiologique) soit son référent ou l'une de ses caractéristiques est assez saillant pour interpeller l'interlocuteur (dans l'exemple le mot Schtroumpf):

Le taxi qui nous emportait vers le paradis était conduit par une sorte de Schtroumpf rose coiffé d'un bonnet en laine multicolore d'où jaillissaient des buissons de cheveux noirs. (G. Brisac, Week-end de chasse à la mère, p. 94)

L'évaluation rapprochée doit être partagée : on accepte comme exemplaire représentatif ou comme approximation (notamment métalinguistique) ce qui est proposé. Mais, de toute façon, compte tenu des marqueurs cotextuels et contextuels précédemment mentionnés qui favorisent l'une ou l'autre interprétation, c'est le bon vouloir de l'interlocuteur d'une part et le degré de connivence «clanique» des locuteurs d'autre part qui vont décider de l'interprétation, à partir d'une structure ambivalente du point de vue de l'analyse grammaticale et de la compréhension sémantique. C'est dans cette optique interlocutive que Noailly (op.cit.) traitait de la désignation médiate déjà mentionnée : elle invoque d'une part le manque d'inventivité du locuteur et la peur de l'incompréhension des interlocuteurs (ou son manque de coopération) face aux motsvalises, créations lexicales ponctuelles et éphèmères. Nos constructions sont, elles, grammaticalisées mais demandent une stratégie du locuteur dans le profil énonciatif dressé afin que celui-ci «parle» à l'interlocuteur, afin que son référent puisse être identifié ou approché.

\section{Conclusions partielles}

Profiler l'énonciation, c'est-à-dire tenter de cadrer le référent afin de jouer sur la connivence du discours, est le rôle tenu par nos trois termes dans les structures en enclosure. La syntaxe, la sémantique et le discursif se trouvent étroitement imbriqués dans leur analyse: le sens du terme, la détermination, la prédication, la reprise anaphorique, l'emploi dans des cadres énonciatifs spécifiques et la réception jouent chacun un rôle dans l'actualisation de l'approximation.

L'accès le plus immédiat au référent n'est pas toujours privilégié, soit par défaut de mot, soit par souci de cerner et de décrire de la façon la plus analytique, soit par connivence affirmée; il y a aussi une motivation expressive, parallèlement à la grammaticalisation 
des termes envisagés, qui débouche sur la métaphore. La médiation de la désignation relève du réglage du sens en discours, dans une dimension interlocutive.

Comme pirouette, nous laisserons à notre lecteur cet exemple curieux qui superpose les critères listés ci-dessus :

Il m'a dit que $M$. de Grignan lui avait parlé d'une espèce de grossesse qui commençait à se faire espérer (Mme de Sévigné, Correspondances t1, p. 246)

Remarquons d'emblée le cadre énonciatif particulier (la lettre permet de supposer une connivence destinateur/destinataire) et la mise en abîme dans le rapport des dires (dire que, parler que), deux situations propices à l'approximation.

Le terme grossesse suppose-t-il des sous-catégorisations? Le dictionnaire donne grossesse gémellaire, trigémellaire, multiple, extra-utérine, ectopique, nerveuse, à risque mais ce sont des termes relevant du vocabulaire médical et non pas de l'usage courant ( $a$ fortiori pour un exemple du XVII ${ }^{\text {ème }}$ siècle).

Mais une grossesse qui «commence à se faire espérer " peut-elle être déjà appelée « grossesse »?

Est-ce quelque chose qui ressemble à une grossesse et pourrait n'en pas être?

Est-ce une grossesse non encore révélée?

Seule la lecture suivie de la correspondance pourra accréditer si cette espèce de grossesse sera bien...une grossesse véritable!

\section{BIBLIOGRAPHIE}

\section{Bibliographie sélective}

BAKHTINE, M./VOLOCHINOV V.N. (1929/1977), Le Marxisme et la philosophie du langage, Paris :

Minuit.

KLEIBER, G. (1990), La sémantique du prototype, Paris : PUF.

LANDHEER, R. (à paraître), « Comment l'espèce se fait une sorte de genre - Quelques observations sur la grammaticalisation de trois classifieurs français : genre, espèce et sorte ", Revue de Sémantique et pragmatique.

MARTIN, R. (1983), Pour une logique du sens, Paris : PUF.

MARTIN, R. (1987), Langage et croyance, Bruxelles : Mardaga.

NOAILLY, M. (1993), « Des divers modes de la coordination nominale, observés dans leur relation aux objets de référence ", Cahiers de Praxématique 21, 72-87.

PERRET, M. (1992), « Or et Ci de référence textuelles » dans M.A. Morel et L. Danon-Boileau, La deixis, Paris : PUF, 579-582.

POTTIER, B. (1992), Sémantique générale PUF : Paris. 
ROSIER, L. (2000), « Les petites rapporteurs de discours » dans Carvalho, P. et Labrune, L. (eds), Grammaticalisation 1. (dé)motivation et contrainte, Travaux linguistiques du Cerlico 13, Presses Universitaire de Rennes.

ROSIER, L. (à paraître), « Genre : le nuancier de sa grammaticalisation » dans Actes du colloque international de Tel-Aviv sur La préposition (septembre 2000), Travaux de Linguistique.

ROSIER, L. (à paraître), « La polysémie des termes genre, style et type », communication au colloque international sur La polysémie, Paris IV/La Sorbonne (17-19 novembre 2000).

ROSIER, L. (à paraître), « Désignation, approximation et médiation du sens », communication au colloque international de Rouen sur La médiation en langue et en discours (6-8 décembre 2000), à paraître dans les actes.

ROSIER, L. (2002), « Sortes d'invectives », Représentations du sens linguistique, D. Lagorgette et P. Larrivée eds., Munich : LINCOM-EUROPA.

TAMBA-MECZ, I. (1981), Le sens figuré, Paris : PUF.

YAGUELLO, M. (1998), Petits faits de langue, Paris : le Seuil.

\section{NOTES}

1. Les exemples sont extraits de la base textuelle Frantext, du corpus Orléans (oral) disponible en ligne via le site LANCOM et d'un corpus personnel.

2. Sur cette question, nous renvoyons notre lecteur à notre article «Genre : le nuancier de sa grammaticalisation » à paraître dans les Travaux de linguistique (actes du colloque de Tel-Aviv sur la préposition).

\section{AUTEUR}

\section{LAURENCE ROSIER}

Université Libre de Bruxelles,

Service de linguistique française et d'analyse du discours 